Revue d'histoire de l'Amérique française

REYUE D.HISTOIRE DE L'AMÉRIQUE FRANÇAISE

MORTON, Suzanne, Ideal Surroundings. Domestic Life in a Working-Class Suburb in the 1920's (Toronto, University of Toronto Press, 1995), 201 p. 17,95 \$

\title{
Veronica Strong-Boag
}

Volume 49, numéro 4, printemps 1996

URI : https://id.erudit.org/iderudit/305476ar

DOI : https://doi.org/10.7202/305476ar

Aller au sommaire du numéro

Éditeur(s)

Institut d'histoire de l'Amérique française

ISSN

0035-2357 (imprimé)

1492-1383 (numérique)

Découvrir la revue

Citer ce compte rendu

Strong-Boag, V. (1996). Compte rendu de [MORTON, Suzanne, Ideal

Surroundings. Domestic Life in a Working-Class Suburb in the 1920's (Toronto,

University of Toronto Press, 1995), 201 p. 17,95 \$]. Revue d'histoire de

l'Amérique française, 49(4), 589-591. https://doi.org/10.7202/305476ar d'utilisation que vous pouvez consulter en ligne.

https://apropos.erudit.org/fr/usagers/politique-dutilisation/ 
MORTON, Suzanne, Ideal Surroundings. Domestic Life in a Working-Class Suburb in the 1920's (Toronto, University of Toronto Press, 1995), 201 p. $17,95 \$$

Les monographies communautaires ont une longue et riche tradition au Canada. Des ouvrages d'un certain âge comme The City Below the Hill de Herbert Ames (1897), St. Denis de Horace Miner (1939) et The People of Hamilton, Canada West de Michael Katz (1975), demeurent des références précieuses. Des additions plus récentes comme Maisonneuve de Paul-André Linteau (1981) et The Gender of Breadwinners de Joy Parr (1990) continuent d'approfondir notre compréhension du passé.

Étant donné qu'elles sont vouées à la reconstitution de la vie quotidienne d'une collectivité, ces études portent une attention plus grande à la classe ouvrière, et, dans une moindre mesure, aux femmes, que la recherche traditionnelle. Elles franchissent également les frontières entre les disciplines en recourant à la sociologie, à l'histoire, à l'anthropologie et à d'autres domaines de recherche pour éclairer les systèmes, les enjeux et les possibilités qui structurent la vie et confèrent prestige et pouvoir. Ce n'est donc pas étonnant que les chercheures féministes aient été particulièrement attirées par cette façon d'aborder le passé.

Ideal Surroundings de Suzanne Morton est une addition valable à la recherche sur les communautés canadiennes. À l'origine une thèse de doctorat en histoire réalisée à l'Université Dalhousie, l'ouvrage traite de façon fluide et instructive de la vie ouvrière dans une municipalité en banlieue d'Halifax après la Première Guerre mondiale. Morton s'inspire entre autres 
de la recherche récente sur les femmes, la classe ouvrière et les provinces de l'Atlantique pour examiner comment les hommes et les femmes de Richmond Heights ont structuré et compris leur vie dans les années 1920. Cette petite ville est une candidate idéale à une recherche en profondeur puisqu'elle a été créée de toutes pièces après l'explosion d'Halifax, et représente la première expérience d'aménagement urbain en milieu ouvrier au Canada.

Morton inverse l'approche du cycle de vie, «one that begins with the elderly and ends with young single women» (p. 13). Le chapitre premier décrit l'apparence de la ville, soulignant sa signification et ses attraits pour les résidants et les urbanistes. Le deuxième chapitre s'attarde aux valeurs dominantes des habitants, c'est-à-dire la «respectabilité» et «le consumérisme», qui étaient fortement démarquées selon le genre principalement en ce qui concerne l'intimité, la propreté, les pensionnaires et l'achat de produits de consommation.

Le troisième chapitre explore la situation des personnes âgées et leurs efforts pour maintenir leur autonomie économique. La perte du rôle de pourvoyeur pour les hommes et de la jeunesse pour les femmes marquait la fin de l'identé de genre pour les deux sexes et rendait les personnes âgées vulnérables à la marginalisation dans une culture de plus en plus centrée sur la jeunesse.

Le chapitre IV examine la vie des couples partagés entre divers discours idéologiques sur le mariage qui mettaient respectivement l'accent sur le partenariat économique, les obligations religieuses ou l'amour romantique. Un puissant impératif domestique motivait «the ideal, and to some extent the practised reality, on an increased home and family centredness in the culture of the respectable working class» (p. 68). Le dilemme des femmes chefs de ménage qui luttaient contre «society's inability to reconcile the role of mother with that of wage-earner» (p. 106) est décrit avec émotion dans le chapitre V.

Le chapitre VI aborde la crise de la masculinité ouvrière traditionnelle confrontée à des identités publiques et privées qui se chevauchaient et parfois se contredisaient. Bien que les hommes de la classe ouvrière soient demeurés plus liés à leur appartenance de classe que les femmes, leur attachement aux privilèges liés à l'idéologie du pourvoyeur traversait les frontières de classe. Le chapitre VII se penche sur les jeunes femmes à la croisée des chemins entre le foyer et le travail salarié. Des vêtements modernes et le travail dans les bureaux et les magasins «fostered a workplace culture of heterosexual romance» (p. 147) qui modifiait la conscience de classe des femmes.

En résumé, Richmond Heights a été témoin, entre les deux guerres mondiales, de la dilution de l'identité de classe, de la complexification des relations hommes-femmes et de la promotion du mythe d'une société sans classes orientée vers la consommation et la domesticité. L'omniprésence de la société de consommation qui a émergé au $\mathrm{XX}^{\mathrm{e}}$ siècle a ultérieurement freiné l'action sociale progressiste au Canada autant que les divisions ethniques et raciales qui sont souvent citées comme cause. 
Malgré ses attraits, Ideal Surroundings n'est pas complètement réussi. En dépit des efforts de Morton pour réunir des voix provenant de différentes sources, le portrait des gens de Richmond Heights reste curieusement partiel. Ils semblent presque désincarnés, des symboles qui servent à appuyer des théories plutôt que des êtres de chair et de sang à mieux connaître. Le problème est fortement lié à la décision de ne pas tenir compte des enfants et de l'enfance sous prétexte que «children were not active participants in the creation of gender ideology, but were more often the intended subjects of socialization» (p. 14). Morton néglige l'importance de l'interaction entre les adultes et les enfants et le pouvoir réel, bien que restreint, qu'avaient les enfants de forger leur propre conscience de classe et de genre. En incluant les enfants et leurs souvenirs, le livre aurait eu accès à une gamme plus vaste d'entrevues ainsi que de matériel biographique et autobiographique, ce qui aurait humanisé davantage les acteurs de Richmond Heights. Malgré ces réserves, Ideal Surroundings constitue une contribution importante à la recherche communautaire et, de manière plus générale, à l'histoire ouvrière et à l'histoire des femmes du Canada.

Centre de recherche en études féministes

et sur les rapports de sexes

Université de la Colombie-Britannique

Traduction: Marie Poirier 